\title{
Synthesis and characterization of poly(ester amide) from renewable resources through melt polycondensation
}

\author{
Y. P. Ge ${ }^{1}$, D. Yuan $^{1}$, Z. L. Luo $^{1}$, B. B. Wang $^{1,2^{*}}$ \\ ${ }^{1}$ School of Materials Science and Engineering, Changzhou University, 213164 Changzhou, China \\ ${ }^{2}$ School of Materials Science and Engineering, North University of China, 030051 Taiyuan, China
}

Received 22 June 2013; accepted in revised form 2 September 2013

\begin{abstract}
Biodegradable poly(ester amide)s (PEAs) were synthesized from lactic acid and 11-aminoundecanoic acid via melt polycondensation. Molecular weights, chemical structures and thermal properties of the poly(ester amide)s were characterized in terms of gel permeation chromatography (GPC), Fourier transform infrared spectroscopy (FTIR), ${ }^{1} \mathrm{H}$ nuclear magnetic resonance ( ${ }^{1} \mathrm{H}$ NMR), differential scanning calorimetry (DSC) and thermogravimetric analysis (TGA), respectively. The PEAs have low molecular weights and display a lower cold crystallization temperature as well as smaller crystallinity by comparison with the pure poly(lactic acid) (PLA). The incorporation of the 11-aminoundecanoic acid into the PLA chain not only improved the thermal stability but changed the decomposition process.
\end{abstract}

Keywords: thermal properties, poly(ester amide)s, polycondensation, characterization

\section{Introduction}

The ecological challenges and the fluctuating petroleum prices have instigated the research and development on the materials from renewable resources to be the hot spot. Nylon 11 and poly(lactic acid) (PLA) have attracted great interest since they can be produced from renewable resources, that is, the beginning material for nylon 11 is castor oil $[1,2]$ and these for PLA can be starch, cane molasses and beet sugar [3, 4]. Poly(ester amide)s (PEAs) are a class of very promising materials combining the favorable properties of polyesters (biodegradability) and polyamides (good mechanical and thermal behavior) [5]. The rigidity due to the double-bond character of the amide group coupled with extensive hydrogen bonding influences the ordering of PEAs and consequently enhances the mechanical and thermal stability. As such, the synthesis of PEAs represents a good alternative to overcome the thermal and mechanical limitations of PLA. Liu et al. [6] had synthesized tough PEAs having short nylon 6 segments in the main chains through polycondensation and chain extension, the initial decomposition temperature of the chain-extended PEAs is over $339^{\circ} \mathrm{C}$. Fonseca et al. [7] synthesized novel PEAs from glycine and L-lactic acid by interfacial polymerization, the method needs to be optimized to afford a higher molecular weight and a narrow molecular weight distribution. Pramanik et al. [8] reported the synthesis of castor oil modified biodegradable PEAs with higher thermal stability. PEA based on caprolactone and 11-aminoundecanoic acid has also been reported by Qian et al. [9]. The same group also synthesized biodegradable PEA based on lactic acid and aminoundecanoic acid and investigated in vitro degradation behaviors [10]. However, PEA with low melting temperatures $\left(\leq 89^{\circ} \mathrm{C}\right)$ were obtained at $50 \mathrm{~mol} \%$ lactic acid loading level or above, which might be attributed the lower molecular weights. In this work, a small quantity of rigid amide group was

\footnotetext{
${ }^{*}$ Corresponding author, e-mail: biaobingwang@hotmail.com (C) BME-PT
} 
distributed into the PLA chains to improve the thermal stability of PLA through melt polycondensation using L-lactic acid (LA) and 11-aminoundecanoic acid as starting materials. The PEAs were characterized by GPC, FTIR, ${ }^{1} \mathrm{H}$ NMR, DSC and TGA.

\section{Experimental}

\subsection{Materials}

L-Lactic acid was supplied as a $90 \mathrm{wt} \%$ aqueous solution by Lingfeng Chemical Reagent (China). 11-aminoundecanoic acid was purchased from Zhonglian Zenong Chemical Co. (China). Tin(II) chloride dihydrate $\left(\mathrm{SnCl}_{2} \cdot 2 \mathrm{H}_{2} \mathrm{O} ; 98 \%\right.$, reagent grade) and p-toluenesulfonic acid monohydrate (TSA; $98.5+\%$, ACS reagent grade) were purchased from Alfa Aesar Co. (America). Other chemical reagents were purchased from Yonghua Chemical (Jiangsu, China).

\subsection{Dehydration/oligomerization}

The lactic acid and 11-aminoundecanoic acid were added to a $250 \mathrm{~mL}$ three-neck flask and stirred with a magnetic stirrer and dehydrated at $110^{\circ} \mathrm{C}$ under atmospheric pressure for $2 \mathrm{~h}$, then at $130^{\circ} \mathrm{C}$ under a reduced pressure of $13300 \mathrm{~Pa}$ for $3 \mathrm{~h}$, and finally at $150^{\circ} \mathrm{C}$ under a reduced pressure of $13300 \mathrm{~Pa}$ for another $4 \mathrm{~h}$. Then a viscous transparent oligomer was obtained, and became solid after cooling to room temperature. Oligomer of pure lactic acid was also synthesized as a control following the same procedures.

\subsection{Polymerization}

The previous flask was equipped with a mechanical stirrer and a reflux condenser that was connected with a vacuum system through a liquid nitrogen cold trap and then mixed with $\mathrm{SnCl}_{2} \cdot 2 \mathrm{H}_{2} \mathrm{O}(0.4 \mathrm{wt} \%$ relative to oligomer) and TSA (an equimolar amout to $\mathrm{SnCl}_{2} \cdot 2 \mathrm{H}_{2} \mathrm{O}$ ) as a binary catalyst. The mixture was gradually heated to $180^{\circ} \mathrm{C}$ with stirring, following which the pressure was reduced gradually to $1330 \mathrm{~Pa}$ in $1.5 \mathrm{~h}$ and the reaction was continued for another $10 \mathrm{~h}$. The product was cooled and dissolved in chloroform and subsequently precipitated into anhydrous ethanol. The resulting solid was filtered and dried under vacuum at $80^{\circ} \mathrm{C}$ for $12 \mathrm{~h}$. Bulk PEAs were labeled as PEA-2.5 and PEA-5 according to the weight ratio of 11 -aminoundecanoic acid to lactic acid. Pure PLA was also synthesized following the same procedures and used as a control.

\subsection{GPC analysis}

The weight $\left(\bar{M}_{\mathrm{w}}\right)$ and number-average molecular $\left(\bar{M}_{\mathrm{n}}\right)$ weight as well as the polydispersity index (PDI, equal to $\bar{M}_{\mathrm{w}} / \bar{M}_{\mathrm{n}}$ ) were determined through GPC (Waters, America). The test was carried out with a Waters 1515 separations module, Waters 2414 refractive index detector. Samples were dissolved in tetrahydrofuran at a concentration of $5 \mathrm{mg} / \mathrm{mL}$. The measurement was performed at room temperature and a flow rate of $1 \mathrm{~mL} / \mathrm{min}$. The molecular weight was calibrated according to a polystyrene standard.

\subsection{FTIR spectroscopy}

FTIR spectra were acquired with an Avatar 370 spectrometer (Thermo Nicolet, America). Spectra were collected in the region of $4000-370 \mathrm{~cm}^{-1}$ with a spectral resolution of $0.1 \mathrm{~cm}^{-1}$.

\subsection{NMR spectroscopy}

${ }^{1} \mathrm{H}$ NMR spectra were acquired at room temperature for PLA and PEAs on an Advance III 400M NMR (Bruker, Rheinstetten, Germany) in $\mathrm{CDCl}_{3}$ containing tetramethylsilane as the internal reference.

\subsection{Thermogravimetric analysis (TGA)}

Decomposition characteristics of the samples were determined with a TG 209 F3 (Netzsch Tarsus, Germany). About $5 \mathrm{mg}$ of each sample was placed in the pan and heated from 50 to $650^{\circ} \mathrm{C}$ at a heating rate of $10^{\circ} \mathrm{C} /$ min under a nitrogen atmosphere.

\section{Results and discussion}

\subsection{Molecular weights}

Molecular weights of PLA and PEAs were measured using GPC, and the corresponding values are summarized in Table 1. As can be seen, the $\bar{M}_{\mathrm{w}}$ of the pure PLA is $25566 \mathrm{~g} / \mathrm{mol}$. By comparison, the molecular weights of the PEAs are decreased with the incorporation of 11-aminoundecanoic acid since it is not easy to amidate under the reaction temperature $\left(180^{\circ} \mathrm{C}\right)$. For example, the $\bar{M}_{\mathrm{w}}$ of the PEA-5 is $16210 \mathrm{~g} / \mathrm{mol}$. The PDI values for the pure PLA and

Table 1. Molecular weights of PLA and PEAs

\begin{tabular}{|l|c|c|c|}
\hline \multicolumn{1}{|c|}{ Sample } & $\overline{\mathbf{M}}_{\mathbf{n}}$ & $\overline{\mathbf{M}}_{\mathbf{w}}$ & $\frac{\mathbf{P D I}}{\left(\overline{\mathbf{M}}_{\mathbf{w}} / \overline{\mathbf{M}}_{\mathbf{n}}\right)}$ \\
\hline PLA & 16230 & 25566 & 1.58 \\
\hline PEA-2.5 & 11889 & 18932 & 1.59 \\
\hline PEA-5 & 11109 & 16213 & 1.46 \\
\hline
\end{tabular}


PEAs range between 1.4 and 1.8, which are similar to those for PLA obtained from the melt polycondensation method $[11,12]$.

\subsection{NMR}

Figure 1 shows the typical ${ }^{1} \mathrm{H}$ NMR spectra of the pure PLA, PEA-2.5 oligomer and corresponding PEA-2.5. The major peaks at 1.58 and $5.17 \mathrm{ppm}$ are assigned to the methyl and methine proton resonances from the PLA main chain. The weak peaks at 4.36 and at $1.28 \mathrm{ppm}$ are assigned to the methine and methyl proton next to the terminal hydroxyl group, respectively. The above two weak peaks could be identified only for LA oligomers [13] since the intensity of these peaks decrease dramatically with the increasing molecular weights. As such, the above
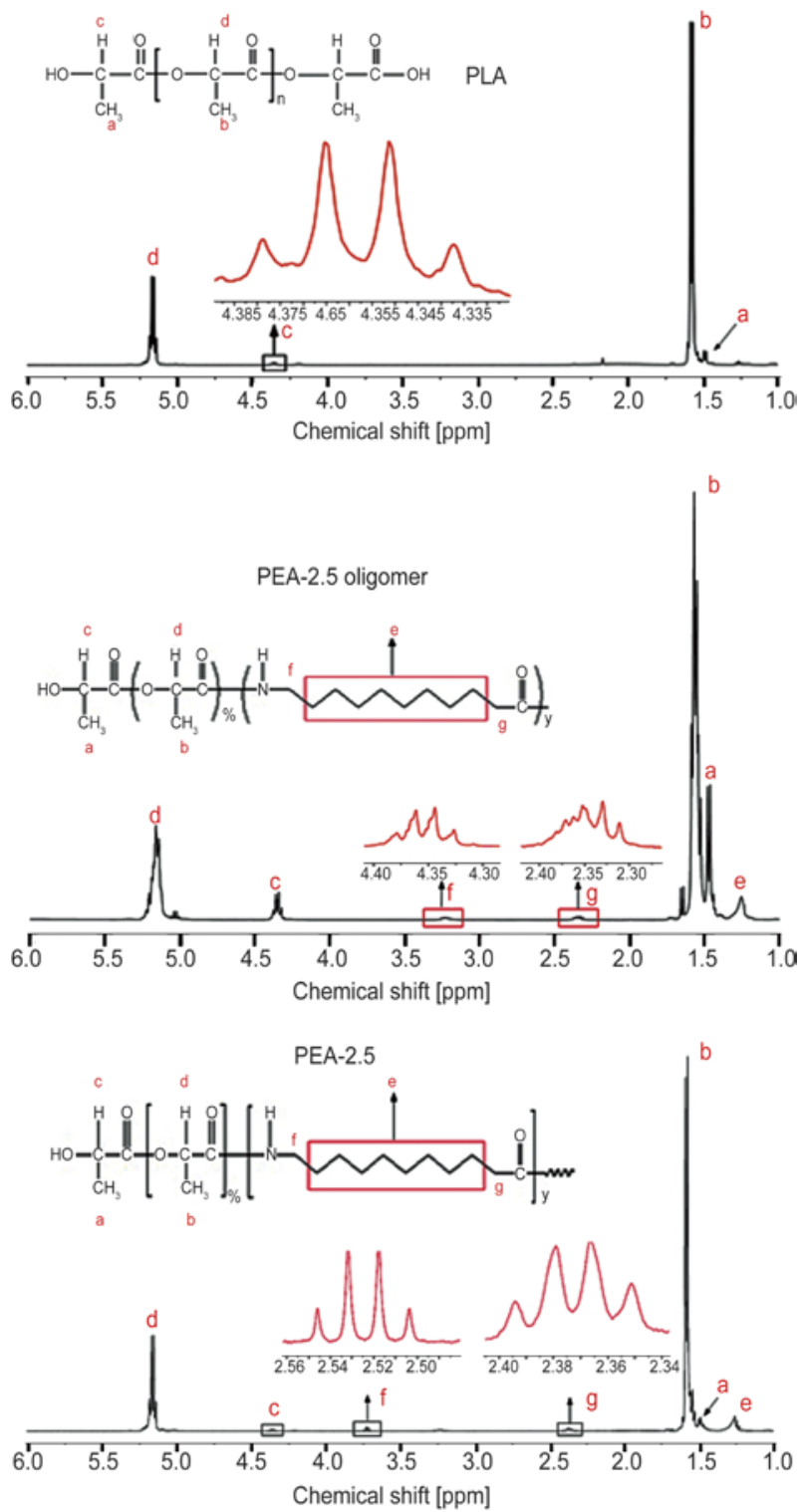

Figure 1. ${ }^{1} \mathrm{H}$ NMR spectra of PLA, PEA-2.5 oligomer and corresponding PEA-2.5 two peaks become obvious in the spectrum of PEA2.5 oligomer. According to the assignment, the degree of polymerization of PEA-2.5 oligomer is estimated to be 8 from the integration ratio between the methane proton of peak $b$ and peak $c$. The spectra of the PEA2.5 oligomer and corresponding PEA-2.5 display three new weak peaks, which corresponds to the methane of 11-aminoundecanoic acid, at $3.22 \mathrm{ppm}$ (peak $\mathrm{f}$ for PEA-2.5 oligomer) or 3.72 ppm (peak $f$ for PEA-2.5), $2.32 \mathrm{ppm}$ (peak g) and $1.25 \mathrm{ppm}$ (peak e), respectively. It is indicating that the aminoundecanoic acid fragment is chemically introduced into the main chain of PLA.

\subsection{FTIR}

The FTIR spectra of PLA and PEAs are illustrated in Figure 2. The pure PLA shows a strong absorption band at $1751 \mathrm{~cm}^{-1}$ corresponding to the stretching vibration of carbonyl groups $(-\mathrm{C}=\mathrm{O})$ from the repeated ester units. The $-\mathrm{C}-\mathrm{O}-$ stretching vibrations from the ester units are observed at 1180,1129, and $1082 \mathrm{~cm}^{-1}$. The bands at 2996 and $2877 \mathrm{~cm}^{-1}$ are assigned to the $-\mathrm{C}-\mathrm{H}$ asymmetric and symmetric stretching vibrations of $\mathrm{CH}_{3}$ groups in the side chains, whereas their bending vibration appears at $1454 \mathrm{~cm}^{-1}$. The band at $2948 \mathrm{~cm}^{-1}$ is attributed to the stretching of $-\mathrm{CH}-$ groups in the main chain of PLA, and its symmetric and asymmetric bending vibrations appear at 1382 and $1358 \mathrm{~cm}^{-1}$. As compared with the pure PLA, the spectra of PEAs display the typical amide peaks at $3304 \mathrm{~cm}^{-1}$ (amide A), $3087 \mathrm{~cm}^{-1}$ (amide $\left.\mathrm{B}\right), 1655 \mathrm{~cm}^{-1}(\mathrm{C}=\mathrm{O}$ stretching, amide I), and $1545 \mathrm{~cm}^{-1}(\mathrm{~N}-\mathrm{H}$ bending, amide II). It is further demonstrating that 11-aminoundecanoic

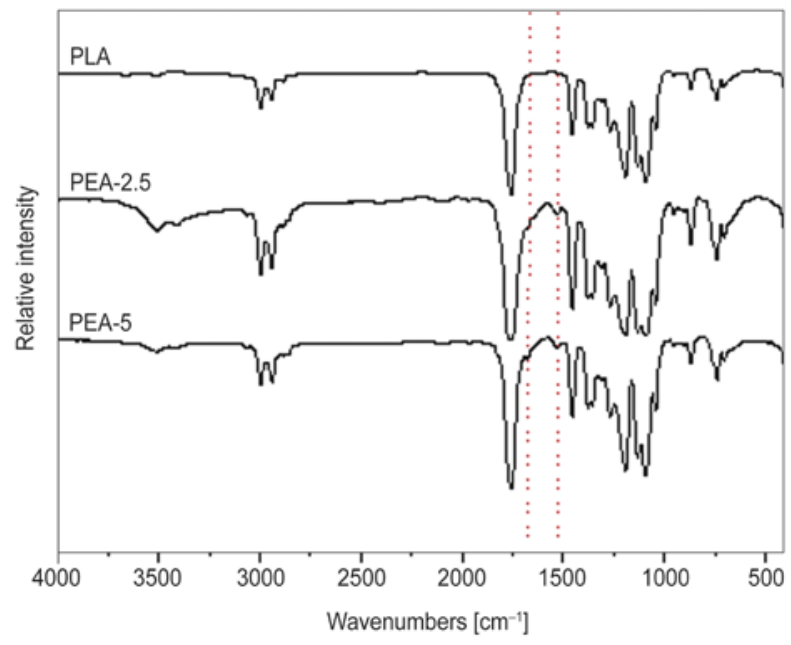

Figure 2. FTIR spectra of PLA and PEAs 
acid fragment is chemically introduced into the main chain of PLA.

\subsection{Thermal properties}

The DSC thermograms (Figure 3) of all samples display glass transition, cold crystallization and melting behaviors, and the quantified results are summarized in Table 2. As can be seen, the glass transition temperature $\left(T_{\mathrm{g}}\right)$ of PEA-5 is about $6^{\circ} \mathrm{C}$ lower than that of the pure PLA because of its lower molecular weight [14]. The PEAs display a lower cold crystallization temperature but far smaller crystallinity than that of the pure PLA. It is attributed to the more readily chain mobility as the reduction of the molecular weights and the irregularity of the PLA chain with the incorporation of the 11-aminoundecanoic acid, respectively. Both PLA and PEAs exhibit double melting behavior, which can be explained by the melt-recrystallization model. The melting temperatures of the obtained PEAs are higher than that of PEAs reported in literature [10] at higher lactic acid contents.

The results of the thermogravimetric analysis of the pure PLA and PEAs are summarized in Table 3, while the weight loss and derivative weight loss curves are presented in Figures 4. The PLA shows a distinct one-stage degradation pattern and reaches

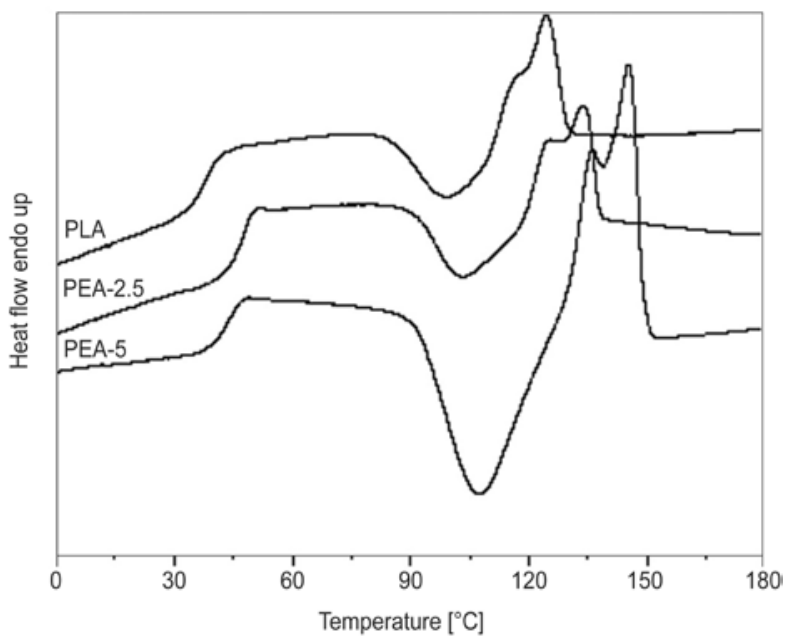

Figure 3. DSC thermograms of PLA and PEAs

Table 2. Thermal properties of PLA and PEAs determined from DSC thermograms

\begin{tabular}{|l|c|r|r|c|c|c|}
\hline Sample & $\begin{array}{c}\mathbf{T}_{\mathbf{g}} \\
{\left[{ }^{\circ} \mathbf{C}\right]}\end{array}$ & $\begin{array}{c}\mathbf{T}_{\mathbf{c}} \\
{\left[{ }^{\circ} \mathbf{C}\right]}\end{array}$ & $\begin{array}{c}\Delta \mathbf{H}_{\mathbf{c}} \\
{[\mathbf{J} / \mathbf{g}]}\end{array}$ & $\begin{array}{c}\mathbf{T}_{\mathbf{m}} \\
{\left[{ }^{\circ} \mathbf{C}\right]}\end{array}$ & $\begin{array}{c}\Delta \mathbf{H}_{\mathbf{m}} \\
{[\mathbf{J} / \mathbf{g}]}\end{array}$ & $\begin{array}{c}\mathbf{X}_{\mathbf{m}} \\
{[\mathbf{\%}]}\end{array}$ \\
\hline PLA & 43.5 & 107.2 & 36.9 & 145.4 & 30.0 & 32.1 \\
\hline PEA-2.5 & 46.8 & 102.7 & 9.8 & 133.9 & 10.6 & 11.3 \\
\hline PEA-5 & 37.7 & 99.7 & 10.5 & 124.6 & 9.6 & 10.3 \\
\hline
\end{tabular}

Table 3. Thermal decomposition temperatures of PLA and PEAs

\begin{tabular}{|l|c|c|c|}
\hline \multicolumn{1}{|c|}{ Sample } & $\begin{array}{c}\mathbf{T}_{\text {onset }} \\
{\left[{ }^{\circ} \mathbf{C}\right]}\end{array}$ & $\begin{array}{c}\mathbf{T}_{\text {end }} \\
{\left[{ }^{\circ} \mathbf{C}\right]}\end{array}$ & $\begin{array}{c}\mathbf{T}_{\max } \\
{\left[{ }^{\circ} \mathbf{C}\right]}\end{array}$ \\
\hline PLA & 214 & 278 & 255 \\
\hline PEA-2.5 & 246 & 378 & $276-335$ \\
\hline PEA-5 & 252 & 480 & $289-340$ \\
\hline
\end{tabular}

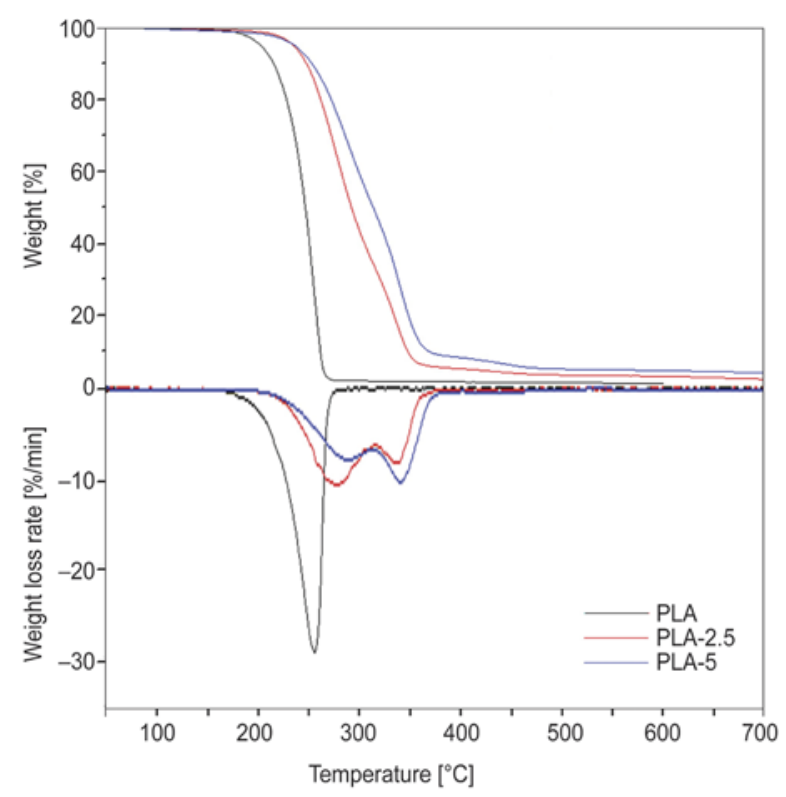

Figure 4. TGA and DTG curves of of PLA and PEAs

its maximum rate at $255^{\circ} \mathrm{C}$. The PEAs, however, display a two-stage degradation pattern. This implies that the degradation mechanism is altered by the incorporation of the 11-aminoundecanoic acid. For PEAs, the first and second weight loss processes belong to the breakage of the ester and amide bond [9], respectively. Moreover, it appears that the incorporation of the 11-amineundecanoic acid into the PLA chain improves the thermal stability. For example, the onset temperatures of PEA-2.5 and PEA-5 are increased by $32 \mathrm{C}$ and $38^{\circ} \mathrm{C}$ as compared to the pure PLA, respectively. The improvement of the thermal stability of PEAs can be attributed to the occurrence of the amide bond which has better thermal stability than ester bond and the intermolecular hydrogen bonding between ester and amide groups.

\section{Conclusions}

PEAs based on lactic acid and 11-aminoundecanoic acid was prepared by melt polycondensation. The lower cold crystallization temperatures and crystallinity of the PEAs were attributed to the reduced molecular weights and the irregularity of the PLA chain due to the incorporation of 11-aminoundecanoic acid. By contrast with the one-stage decom- 
position process of the pure PLA, the PEAs displayed two-stage decomposition process. Moreover, the thermal stability of the PEAs was higher than that of the pure PLA.

\section{Acknowledgements}

This work was financially funded by the Priority Academic Program Development of Jiangsu Higher Education Institution and Qing Lan project of Jiangsu.

\section{References}

[1] Wang B-B., Hu G-S., Zhao X., Gao F-Z.: Preparation and characterization of nylon 611 copolymer. Materials Letters, 21-22, 2715-2717 (2006).

DOI: $10.1016 /$ j.matlet.2006.01.076

[2] Wan J., Bu Z-Y., Li C., Fan H., Li B-G.: Preparation, melting, glass relaxation and nonisothermal crystallization kinetics of a novel dendritic nylon-11. Thermochimica Acta, 524, 117-127 (2011).

DOI: $10.1016 /$ j.tca.2011.07.002

[3] Ishimoto K., Arimoto M., Okuda T., Yamaguchi S., Aso Y., Ohara H., Kobayashi S., Ishii M., Morita K., Yamashita H., Yabuuchi N.: Biobased polymers: Synthesis of graft copolymers and comb polymers using lactic acid macromonomer and properties of the product polymers. Biomacromolecules, 13, 3757-3768 (2012).

DOI: $10.1021 / \mathrm{bm} 301212 \mathrm{a}$

[4] Liao C-C., Wang C-C., Chen C-Y.: Stretching-induced crystallinity and orientation of polylactic acid nanofibers with improved mechanical properties using an electrically charged rotating viscoelastic jet. Polymer, 52, 4303-4318 (2011). DOI: $10.1016 /$ j.polymer.2011.07.031

[5] Mallakpour S., Zadehnazari A.: Advances in synthetic optically active condensation polymers-A review. Express Polymer Letters, 5, 142-181 (2011). DOI: $10.3144 /$ expresspolymlett.2011.15

[6] Liu S., Li C., Zhao J., Zhang Z., Yang W.: Synthesis and characterization of polyesteramides having short nylon- 6 segments in the main chains through polycondensation and chain extension. Polymer, 52, 60466055 (2011).

DOI: $\underline{10.1016 / j . p o l y m e r .2011 .10 .048}$
[7] Fonseca A. C., Serra A. C., Coelho J. F. J., Gil M. H., Simões P. N.: Novel poly(ester amide)s from glycine and L-lactic acid by an easy and cost-effective synthesis. Polymer International, 62, 736-743 (2013). DOI: $10.1002 /$ pi.4356

[8] Pramanik S., Sagar K., Konwar B. K., Karak N.: Synthesis, characterization and properties of a castor oil modified biodegradable poly(ester amide) resin. Progress in Organic Coatings, 75, 569-578 (2012). DOI: 10.1016/j.porgcoat.2012.05.009

[9] Qian Z., Li S., He Y., Li C., Liu X.: Synthesis and thermal degradation of biodegradable polyesteramide based on $\varepsilon$-caprolactone and 11-aminoundecanoic acid. Polymer Degradation and Stability, 81, 279-286 (2003).

DOI: 10.1016/S0141-3910(03)00098-3

[10] Qian Z., Li S., Zhang H., Liu X.: Synthesis, characterization and in vitro degradation of biodegradable polyesteramide based on lactic acid. Colloid and Polymer Science, 281, 869-875 (2003).

DOI: $10.1007 / \mathrm{s} 00396-002-0846-1$

[11] Moon S. I., Lee C. W., Miyamoto M., Kimura Y.: Melt polycondensation of L-lactic acid with $\mathrm{Sn}$ (II) catalysts activated by various proton acids: A direct manufacturing route to high molecular weight poly(L-lactic acid). Journal of Polymer Science Part A: Polymer Chemistry, 38, 1673-1677 (2000).

DOI: 10.1002/(SICI)1099-0518(20000501)38:9<1673 :aid-pola33>3.0.CO;2-T

[12] Moon S. I., Kimura Y.: Melt polycondensation of Llactic acid to poly(L-lactic acid) with $\mathrm{Sn}$ (II) catalysts combined with various metal alkoxides. Polymer International, 52, 299-305 (2003).

DOI: $10.1002 /$ pi.960

[13] Espartero J. L., Rashkov I., Li S. M., Manolova N., Vert M.: NMR analysis of low molecular weight poly (lactic acid)s. Macromolecules, 29, 3535-3539 (1996). DOI: $10.1021 / \mathrm{ma} 950529 \mathrm{u}$

[14] Jamshidi K., Hyon S-H., Ikada Y.: Thermal characterization of polylactides. Polymer, 29, 2229-2234 (1988). DOI: 10.1016/0032-3861(88)90116-4 